\title{
Experimental Progress in Deep and Broadband Infrared Nulling for TPF-I
}

\author{
J. Kent Wallace ${ }^{1}$, Randall Bartos, Robert Gappinger \\ Frank Loya, Steve Moser, and John Negron \\ ${ }^{1}$ Jet Propulsion Laboratory, California Institute of Technology, 4800 Oak Grove Dr., \\ Pasadena, CA 91109, USA \\ email: James.K.Wallace@jpl.nasa.gov
}

\begin{abstract}
Our activities are focused upon the experimental demonstration of deep nulling in the mid-IR over a wide bandpass. Specifically, our near-term goal is demonstrating a contrast of $10^{-6}$ at $10 \mu \mathrm{m}$ with a $25 \%$ spectral bandwidth. To meet this goal, several areas of technology development are required. These include: single-mode infrared fibers, bright infrared sources, laser path-length and tip/tilt metrology, and improvements to detection sensitivity. Progress in each of these areas of technical development will be reviewed as well as their impact on the overarching technical milestones.
\end{abstract}

Keywords. techniques: interferometric, instrumentation: detectors, infrared: general

\section{Introduction}

The challenges for an infrared nulling interferometry mission can be broken down into the ability to control systematic errors and the ability to deeply null over a broad spectral bandpass. This paper will describe the technology development that is a requirement for achieving deep and broadband nulls in the mid infrared independent of both the nulling architecture and the systematic errors.

\section{Nulling Requirements}

Our specific testbed requirements are driven entirely by a flow down of requirements that in turn are driven by our core science objectives, namely number of target stars. This allocation of factors that contribute to the ultimate achievable null contrast is outside the scope of this paper. This topic has been covered in great detail previously (Noecker, Lay, Ware \& Dubovitsky (2003)). Our testbed is tasked with the experimental demonstration of deep nulls that are consistent with the objectives of the mission. The achromatic nulling testbed must demonstrate non-cryogenic nulling to a level of $10^{-6}$ for a thermal source of bandwidth $>25 \%$ in the 8 to $12 \mu \mathrm{m}$ region. This null is to be stable to an order of magnitude greater than the average null. For each of these architectures, deep nulling would not be possible without the development of a bright source, sensitive detector, mid-IR single mode filter and laser metrology for path-length stabilization. That is, deep contrast will only be achieved by a combination of the nulling architectures and the technical developments described herein.

The typical IR thermal source is a resistive element that has a blackbody temperature of about $1500 \mathrm{~K}$. Achieving more photons in the mid IR is a challenge because as the temperature of a blackbody is increased, most of the gain in flux occurs at the shorter wavelengths in the visible portion of the spectrum. The flux at a wavelength of $10 \mu \mathrm{m}$ grows almost linearly with temperature. Thus, in order to increase the flux by a significant 
amount, say an order of magnitude, the effective temperature must increase by almost an order of magnitude: $10,000 \mathrm{~K}$. We will describe the construction of a source with this effective temperature.

The noise floor of the detector limits the achievable dynamic range at null. Clearly dynamic range is also improved by not only going higher with the source but by going lower with the noise floor of the detection process. The current detector is a single pixel $\mathrm{HgCdTe}$ cooled to liquid nitrogen temperatures. One of the most sensitive detectors currently available is a single pixel of a MidIR array. These arrays require a more complicated arrangement: liquid helium operating temperatures, low thermal background environments, and a set of read out electronics to allow slow, full-frame operation as well as rapid readout of a few pixels. Our design for such a system and its current status will be described below.

Two wavefront errors must also be addressed in order to reach deep nulls. The cumulative effect of surface figure errors throughout the beam train serves not only to degrade the imaging quality it also prevents complete interference at the point of recombination. This can be mitigated to some degree by using optics of extremely good wave front quality, or by spatially filtering the wave front before detection. Its not an oversimplification to say that spatial filtering not only reduces the requirements on surface figure errors for the optics, but it makes deep nulling possible. Deep and broadband null would be unreachable were it not for single mode optical filters (fibers). Our solution to this vexing technical problem will be described.

Path length fluctuations due to mechanical vibrations in the experiment, or differential index of refraction fluctuations of air in the two arms of the interferometer will degrade nulls. These path length fluctuations can be monitored and stabilized with a laser metrology system that is independent of the nuller itself and only acts to make rigid the path length differences at the point of recombination. Computing power matured to the point where closed loop operation of a few hundred hertz and data recording at $10 \mathrm{kHz}$ is now feasible.

\section{Nulling Technologies}

\subsection{Arc Source}

Increasing the effective temperature is the only way to get a sufficient quantity of $10 \mu \mathrm{m}$ photons for a blackbody radiator. We developed a source that was based upon the work of very similar source at NIST (Bridges \& Migdal (1996)). Typically, arcs are quite bright in the visible portion of the spectrum, however at $10 \mu \mathrm{m}$, the source is not optically thick. A design whereby its possible to look along the length of the arc increases the optical depth and emissivity.

Our design made fundamental improvements to the previous designs (McIntosh, Wofford, Lucchese \& Bevan (2001)) namely: improving the flow of chilled water through the housing, active cooling of the electrodes and creating a manifold for the distribution of argon gas. The source also employs a few additional safety features such as a temperature sensor on the body of the source, and a water flow meter for the chilled water supply. Both are interfaced to the electrical interlock such that a failure of either will shut the current source down. The system is also enclosed with a housing that makes accidental shorting of the electrodes virtually impossible. The system readily strikes with a tungsten welding rod. At peak operating conditions the systems consumes $8 \mathrm{~kW}$ of electrical energy steady-state. The increase in flux from arc source is shown in figure 1 . 


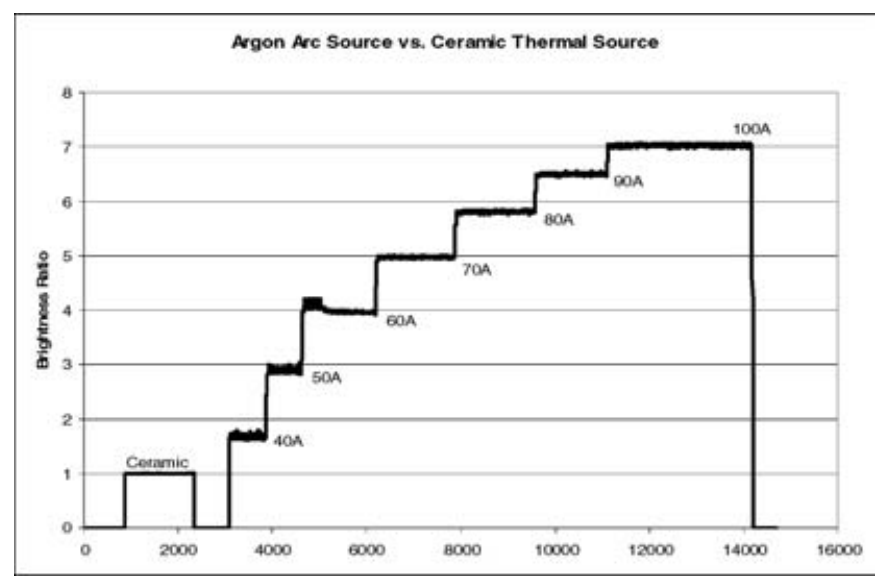

Figure 1. The improvement in IR flux from an argon arc source in comparison to the typical resistive element.

\subsection{MidIR Camera}

The detector of choice for improving the sensitivity of our null measurements is the arsenic-doped silicon, block-impurity band high-flux array from DRS Technologies. This device is $128 \times 128$ in size with 75 um pixels. If operates at a temperature of $10 \mathrm{~K}$ and needs extensive baffling of the thermal background to minimize signal corruption. The dewar has several conveniences. An integral liquid cryogen level sensor for both the LN2 and the LHe will be used in conjunction with the autofill system for both the liquid cryogens. The system is also outfitted with adjustments external to the dewar that permit adjustment of the location of the PSF on the array by pivoting the whole dewar about a point that is also the center of the entrance pinhole. The LHe cold finger also provides a thermal contact to a cold baffle also at LHe temperatures. This provides a very cold solid angle to minimize thermal background. Quick and simple access to the optical mounting surface is accomplished by first removing the bottom dewar housing and then the inner cold shield. Since the electrical feedthroughs are located on the opposite side of this dewar split, there is no additional complication from disconnecting the fanout board. The optical beam train subsequent to the pinhole includes a re-collimating lens, a bandpass filter and low-order direct view prism followed by another lens that forms the image on the array. The array temperature is maintained by temperature sensor and heating element. A total of ten temperature sensors are placed at different locations along the beam train and on the cold plate. Two additional KF-40 flanges are also available in the event that more feedthroughs may be necessary.

\subsection{Single Mode Spatial Filters}

Single mode fibers have long be known for their ability to extract from a distorted wave front its underlying, perfect component (Mennesson, Ollivier \& Ruiller (2000)). It is for this ability (to modally filter the output of an optical system) that we view these devices as absolutely essential in allowing us to demonstrate deep nulling. However, it should also be mentioned that a couple of nulling architectures rely upon a single mode input source in order to reach highest possible contrast. Both the through-focus and nulling periscopes require a single mode input source. These architectures perform a geometric flip of the pupil such that at the point of recombination, opposite sides of the pupil are interfering with each other. The only way to insure that this occurs completely is to have an input single mode source whose phase relationship with other parts of the pupil plane 


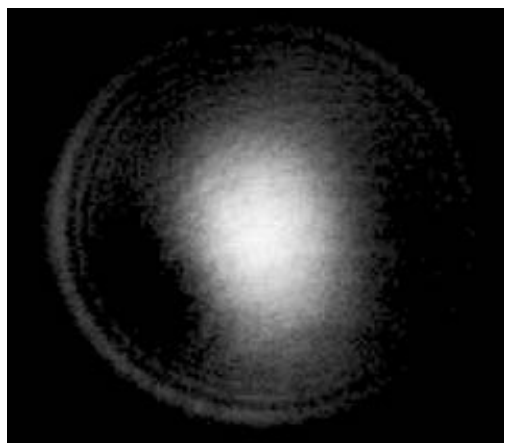

Figure 2. Far field intensity distribution of single-mode chalcogenide Mid IR fiber.

is constant. The spatial filters created for our testbed appear to be single mode fibers. These were procured via a subcontract with Naval Research Laboratories (NRL). Their double-crucible technique for creating near-IR fibers is well documented (Sanghera \& Aggarwal (1998)). With a slight change in the glasses that form the core and cladding, they produced fibers which appear to be single mode.

The single mode behavior is predicted based upon a direct measurement of the index of refraction of the core and cladding glasses as well as the geometric radius of the core. The fibers are conveniently connectorized and packaged in a protective jacketing. The intensity distribution in the pupil plane using a single mode fiber (as measured in our experimental setup) is shown in 2. More extensive testing to characterize their single mode behavior is on-going.

Unfortunately, these fibers suffer from large Fresnel losses at each air/glass interface. The glasses employed have a high index of refraction: the result is that after passing through two fibers, the throughput is only $40 \%$ of what it would be without them.

\subsection{Laser path-length metrology}

The path length stabilization schemes for the Mid IR require a measurement wavelength that is proxy to the science band to stabilize the optical path internal to the interferometer. Fortunately for us, these laser metrology systems for path length stabilization have a long and fruitful history in interferometry. Our initial attempts at path length stabilization have permitted us to demonstrate sub-nanometer control. This level of control is consistent with our ultimate nulling milestone of one million to one. Unfortunately, piston stability is only part of our technical challenge. When coupling into a single mode spatial filter, point errors couple in as amplitude errors. Therefore tip/tilt metrology is required to maintain a constant angular alignment between the two arms of the interferometer. This angular metrology is accomplished by introducing another pair of linear metrology beams that are collinear with the original pencil beam for piston control. The signals from the three gauges then form a metric for piston, tip and tilt. We have built up a test station where we can test and develop the full tip/tilt/piston metrology system. It is wrapped around a compact nulling interferometer working at $10 \mu \mathrm{m}$. This will allows us to fully explore its behavior before integrating it with the rest of the nulling architectures.

\section{Conclusions}

Several areas of technology development are required in order to experimentally demonstrate deep nulling of mid IR light over a broad optical bandpass. We have described: 1) 
an argon arc source, 2) a mid infrared camera, 3) single mode spatial filters and 4) laser metrology for path length stabilization. We have made great strides in bringing all of these into reality and are beginning the process of integration. With all of these elements working in concert, we expect to experimentally demonstrate the nulling consistent with requirements for NASAs TPF-I.

\section{Acknowledgements}

The work described in this paper was carried out at the Jet Propulsion Laboratory, California Institute of Technology, under contract with the National Aeronautics and Space Administration and supported by the Terrestrial Planet Finder Program TPF.

\section{References}

Bridges, J.M. \& Migdal, A.L. 1996, Metrologia 32, 625

McIntosh, A.L., Wofford, B.A., Lucchese, R.R., \& Bevan J.W. 1991, Infrared Physics \& Technology 42, 509

Mennesson, B., Ollivier, B., \& Ruiller, C. 2000, J. Opt. Soc. Am. 19, 3, 596

Noecker, M.C., Lay, O.P., Ware, B., \& Dubovitsky, S. 2003, Proc SPIE 5170, 79

Sanghera, J.S. \& Aggarwal, I.D. 1977 in: J.S. Sanghera and I.D. Aggarwal (eds.), Infrared Fiber Optics (CRC Press Inc.), p. 325 

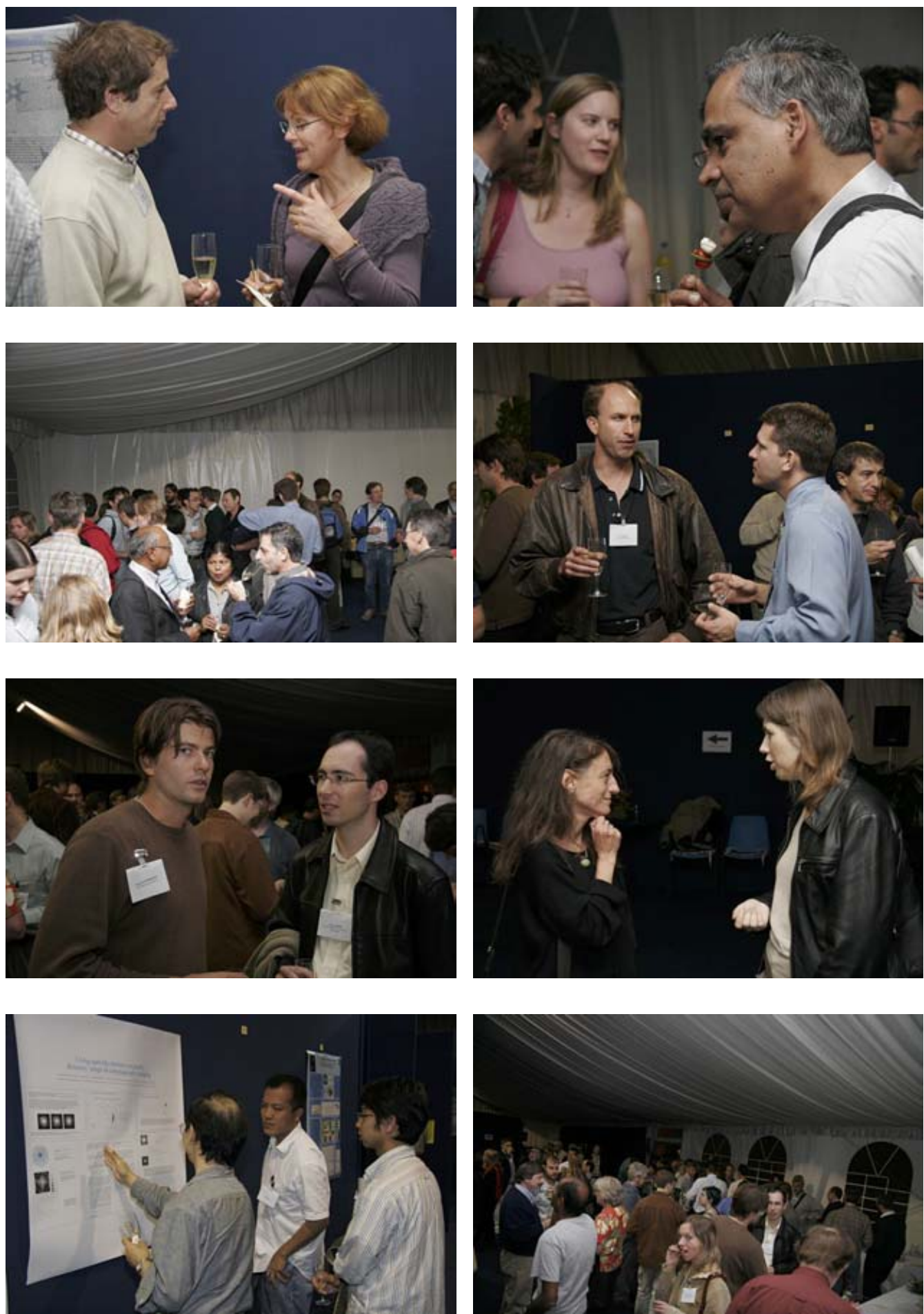

All photographs: Laurent Thareau [1.thareau@free.fr]. 